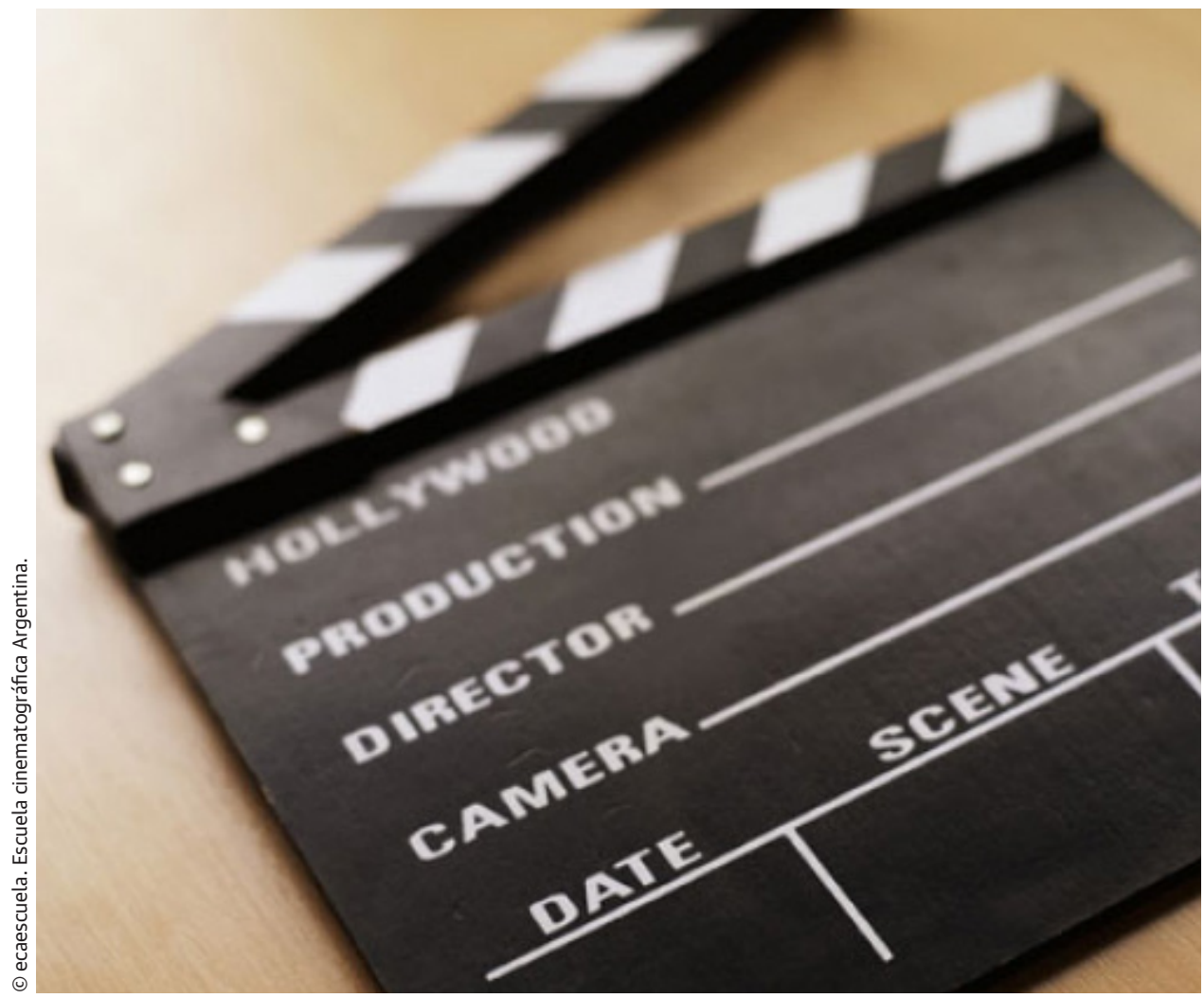

\title{
LA ENSEÑANZA OFICIAL DE CINE EN ESPAÑA
}

\section{OFFICIAL FILMMAKING EDUCATION IN SPAIN}

Lucio Blanco Mallada / mallada@ucm.es

UNIVERSIDAD COMPLUTENSE DE MADRID 


\section{RESUMEN}

En este artículo se recoge la historia de la enseñanza oficial de cine en España: los dos centros en los que se llevó a cabo, sus sedes, sus directores y las prácticas de fin de carrera. Todo ello visto desde la división en tres etapas del tiempo de existencia de la enseñanza anterior a su incorporación a la universidad: la etapa fundacional, la edad de oro y la crisis que lleva al cierre. A lo largo de estas tres etapas hubo un cambio de actitud en el alumnado, desde el entusiasmo y la ilusión inicial al desencanto y rechazo absoluto hacia la Escuela en los últimos años.

PALABRAS CLAVE Cine español, Escuela Oficial de Cinematografía, enseñanza, Filmoteca Española

\section{ABSTRACT}

This article discusses the history of official filmmaking studies in Spain: both centers were it was taught, their headquarters, headmasters and their senior projects. This shall be addressed by dividing into three stages the time during which this education was available, prior to its incorporation into university degrees: The foundational stage, the golden age and the crisis that leads to their closure. During these three stages, their students underwent a change in their attitude, from their initial enthusiasm and excitement to disenchantment and the absolute rejection of their school during its final years.

KEYWORDS

Spanish Cinema, Escuela Oficial de Cinematografía, teaching, Filmoteca Española 


\section{INTRODUCCIÓN}

El Instituto de Investigaciones y Experiencias Cinematográficas -IIEC-, fundado en 1947 y posteriormente denominado Escuela Oficial de Cinematografía -EOC- representa uno de los capítulos más desconocidos de la historia del cine español. Aunque existe una amplia documentación y se conserva un inmenso material inédito, son pocos los trabajos que se han realizado sobre ella. El primero es la tesis doctoral de Lucio Blanco (1990), realizada en la Universidad Complutense de Madrid y dirigida por el doblemente egresado de la escuela, Luis Enrique Torán. Existen posteriores investigaciones sobre la escuela como las abordadas por Asier Aranzubia Cob y José Luis Castro de Paz (2010) -que se ocupa de la práctica de Luciano - o los trabajos de Luis Deltell sobre algunos egresados, como Antonio Lara (2009), Josefina Molina (2015) y Juan Antonio Porto (2014). Por tanto, poco se ha investigado sobre el desarrollo administrativo de este centro académico.

En este artículo nos proponemos configurar una categorización fijando las tres etapas fundamentales de la institución como un primer acercamiento global a lo que representó dicha institución en la cultura española.

\section{LAS TRES ETAPAS}

Los estudios oficiales de cinematografía, hasta el nacimiento de la Facultad de Ciencias de la Información -inaugurada en 1971-, se impartían en el IIEC y, posteriormente, en la EOC. Estos dos últimos se pueden considerar como dos centros distintos, dadas las características tan diferenciadas entre ambos y las circunstancias tan dispares que se dieron entre uno y otro. Aunque lo más habitual, y lo que haremos en este artículo, es considerar al IIEC y a la EOC como un mismo centro que evoluciona a la par que el contexto social y que termina siendo completamente diferente al inicial.

En el periodo de existencia de este sistema de estudios preuniversitario se dan tres etapas: la primera, que comenzó desde su fundación en
1947, era casi mística ${ }^{1}$, como la describen los que la vivieron. Esta fase pionera nació con el fervor cinematográfico ${ }^{2}$, propia del carácter innovador de su tiempo, con el que se compensaba la carencia de medios. La vocación, el entusiasmo y las ganas de constituir el IIEC, tanto de profesores como de alumnos, lograron imponerse a todo tipo de problemas. "No había dinero y no importaba” afirmaba Martín Patino ${ }^{3}$ explicando la mentalidad de aquella época. El sentido casi apostólico de este período fundacional entra en crisis a mediados de los años cincuenta.

Una segunda etapa se caracterizó por la consolidación de los estudios y del centro. Todos los alumnos lo recuerdan como la edad de oro de la escuela, en la que se logró lo imprescindible para que la obra iniciada pudiese llegar a su meta: edificio propio, más medios, cátedras especializadas y exhibición pública de las prácticas de fin de carrera de los egresados, hecho este último que influyó notablemente en la incorporación de los titulados a la industria cinematográfica. Aunque lo realmente decisivo para el reconocimiento de este centro de estudios fue la sinergia que se creó al coincidir José Luis Sáenz de Heredia -de gran prestigio en la industria- como director del centro y José María García Escudero como Director General de Cinematografía. Este impulso terminó fraguando el llamado Nuevo Cine Español.

Finalmente, existe una última fase en la que, a pesar de contar con mejores medios técnicos, el centro entra en una crisis permanente. El conflicto político, administrativo y estudiantil es sistemático y la pérdida de interés por parte del alumnado es palpable ${ }^{4}$. Los discentes y la direc-

1 Entrevista de Lucio Blanco Mallada a Basilio Martín Patino el día 07-10-1984.

2 Ibídem.

3 Ibídem.

4 Rodríguez Merchán aborda el cierre de la siguiente manera: "La bonanza económica y cierta sensibilidad política hacia la mejora de la educación superior en España permite a la Escuela disponer de mejores equipos y de más medios que nunca. Sin embargo, esta situación es una especie de canto del cisne. Pese a que los alumnos logran rodar prácticas más profesionales, incluso en ocasiones encargadas por la propia industria, la sentencia 
ción del centro se enfrentan de un modo irreversible y esto conduce al cierre de la EOC (Gómez, 2002: 204).

A pesar de la clausura de la escuela, los estudios de cine adquieren rango universitario con el nacimiento de la Facultad de Ciencias de la Información. La apertura de este centro no obligaba al cierre de la EOC, que podría haber seguido existiendo como una institución más orientada a la formación profesional especializada, como ocurrió con la Escuela de Turismo o con el Instituto RTVE. Las causas de la extinción fueron el deterioro que había sufrido debido al cambio de mentalidad del alumnado, el enfrentamiento abierto entre estos y la dirección y el desdén mostrado por la industria hacía estos estudios.

\section{LOS ANTECEDENTES}

En 1941 se desarrolló en la Escuela de Ingenieros Industriales un curso de cinematografía con carácter puramente técnico, donde se trataban cuestiones de electroacústica y sensitometría. Esta enseñanza era impartida por Vitoriano López García ${ }^{5}$, futuro Director del IIEC. Debido al éxito de estas lecciones se creó la asignatura de Electroacústica en la carrera de Ingeniería Industrial.

Un poco más tarde, en 1945, se celebró en la Facultad de Filosofía y Letras un ciclo de conferencias con el título "Iniciación Cultural Cinematográfica" que se orientaba hacia el sentido artístico y cultural del cine. Los promotores de esta actividad se unieron con Victoriano López García para plantear la creación de un instituto

de muerte del centro está ya dictada. Considerado como uno de los múltiples nidos de rojos que proliferan entre la joven intelectualidad española, los políticos han decidido cerrar la Escuela de Cine de manera definitiva" (Rodríguez Merchán, 2007: 15).

5 Existe una entrevista a Victoriano López García en la que relata cómo surgió el curso de cinematografía que se impartió en la Escuela de Ingenieros Industriales. Donde daba clase con un libro, Lecciones de cine. Además, intervenía, junto con Victoriano López García, Luis Marquina, el hijo del conocido dramaturgo (Pozo, 1984: 76). de cinematografía inspirado en el Centro Sperimentale di Cinematografia que ya existía en Italia.

\section{EL CENTRO DE ESTUDIOS OFICIALES DE CINEMATOGRAFÍA}

\subsection{Legislación}

El IIEC se crea por las siguientes órdenes ministeriales: Ministerio de Educación Nacional, de 16 y 18 de febrero de 1947, confirmadas por un Decreto de la Jefatura del Estado de 18 de mayo de 1951 (Sánchez Biosca, 2007: 89-110). Cambia su nombre por una Orden Ministerial de 8 de noviembre de 1962, Ministerio de Información y Turismo, pasando a llamarse Escuela Oficial de Cinematografía (Aranzubia Cob y Castro de Paz, 2010: 17).

Desaparece por aplicación de la Ley General de Educación y Financiación y Financiamiento de la Reforma Educativa de 1976 que, en el apartado 5 de la Disposición Transitoria $2^{\mathrm{a}}$, dice: "Los estudios de Periodismo y demás medios de comunicación se incorporarán a Educación Universitaria... sin perjuicio de aquellas (enseñanzas) que solo requieran la capacitación que otorga la Formación Profesional en cualquiera de sus grados”. Estas últimas palabras dejaban abierta la posibilidad de que la EOC pudiera seguir existiendo por la vía de la formación profesional, opción que, como se indicó, fue seguida por las Escuelas de Turismo y de Radio y Televisión.

La EOC era, desde hacía ya años, un centro conflictivo e incómodo para la administración. La salida de José María García Escudero de la Dirección General de Cinematografía supuso la liquidación de la política cinematográfica que este había sostenido y el fin de la escuela. La industria cinematográfica nunca había mostrado interés en la oferta que proporcionaba este centro, salvo en el periodo en el que estuvo Sáenz de Heredia como director, debido a la influencia personal de este cineasta más que al buen hacer de la EOC en sí. La Administración dejó de tener interés en la Escuela con el cambio de gobierno que se produjo a principios de los años sesenta. 


\subsection{Las sedes}

Desde 1947 hasta 1958, el IIEC se estableció en la Escuela de Ingenieros Industriales, que le prestó una parte de sus locales. Unos años antes, desde 1954 a 1955, el alumnado mantuvo una huelga con carácter únicamente académico, sin ninguna reivindicación política, en la que solo se pretendía conseguir más medios y mejores condiciones. Tras la sustitución de Vitoriano López García en la dirección del IIEC, la Escuela de Ingenieros Industriales reclamó al instituto los locales que les estaban cediendo. Esta situación provocó una nueva huelga estudiantil, el cambio de director y el cierre de la institución durante algunos meses.

Durante el curso 1959-60, se albergó el instituto en la sede de Fomento de las Artes y las Letras. La tercera sede se ubicó en Cine Arte, lugar que el alumnado recuerda como adecuado y práctico, como explicaba Claudio Guerín Hill: "Cine Arte era una escuela. Yo tengo de ella un recuerdo feliz" (Aranzubia Cob y Castro de Paz, 2010: 17). Tras esta etapa se instaló en el antiguo edificio del Ministerio de Información y Turismo, donde permaneció desde el curso 1960-61 hasta el 1966-67.

Finalmente se inaugura un edificio propio en la carretera de la Dehesa de la Villa, pero con esta sede llegará también la crisis del centro y su cierre definitivo. Este espacio fue el primero en el que, por dos cursos, se alojó la Facultad de Ciencias de la Información de la Universidad Complutense. Paradójicamente este edificio, dotado de todo lo necesario, no satisfizo al alumnado. Incluso, de algún modo, fue una de las causas del fin de la EOC. Así lo recuerda Manuel Revuelta: "En el fondo es la muerte de la escuela. Monte Esquinza era un centro de reunión de gente, nos reuníamos allí principalmente para ver películas y en el momento del paso a la Ciudad Universitaria se perdió todo eso... Se convirtió en un centro académico, frio, distante...”。.

6 Entrevista de Lucio Blanco Mallada a Manuel Revuela Moreno el día 12-05-1985.

\subsection{Los directores}

La dirección del centro fue encomendada a las siguientes personas:

- Vitoriano López García, desde su fundación en 1947 hasta el 30 de septiembre de 1955.

- José María Cano Lechuga, desde el 30 de septiembre de 1955 al 4 de marzo de 1959.

- José Luis Sáenz de Heredia, desde el 14 de marzo de 1959 al 21 de octubre de 1963.

- Luis Ponce de León Ronquillo, desde el 31 de octubre de 1963 la 1 de julio de 1964.

- Carlos Fernández Cuenca, desde el 1 de julio de 1964 al 22 de febrero de 1968.

- Antonio Cuevas Puente, desde el 1 de julio de 1968 al 1 de mayo de 1969.

- Juan Julio Baena Álvarez, desde 25 septiembre de 1969 hasta la extinción del centro.

El periodo de Vitoriano López García es, como vimos, en donde se manifiesta la ilusión y la vocación cinematográfica de estudiantes y docentes por encima de pasiones ideológicas y de carencias económicas. No hay quejas ni del director hacia sus alumnos ni de estos hacia él. Fue sustituido inexplicablemente tras la huelga que mantuvieron los estudiantes en demanda de más y mejores medios. Quizá el deterioro de las relaciones entre la Escuela de Ingenieros Industriales y el IIEC obligó a la Administración a cesarle.

José María Cano Lechuga, en cambio, fue muy poco apreciado por el alumnado. Fue tomado como un censor y un "comisario político"7 por la mayoría de los egresados. José Luis Sáenz de Heredia fue el siguiente en hacerse cargo de la dirección del centro en un momento difícil. Su nombramiento se hizo a propuesta de los estudiantes a la Dirección General de Cinematografía. La Administración aceptó esta petición sin ninguna vacilación. Sáenz de Heredia no politizó la enseñanza y actuó de forma tolerante y democrática. La confianza en él por parte de la administración y su prestigio entre la industria cinematográfica hicieron que en el centro se viviera la edad de oro: nuevo local, comienzo de la

7 Entrevista de Lucio Blanco Mallada a Fernando Sánchez Dragó el día 21-08-1985. 
construcción del edificio definitivo en un solar de propiedad del centro, mejora de las retribuciones al profesorado, creación de las cátedras y exhibición pública de las prácticas de fin de carrera seguidas con interés por un buen número de profesionales de la industria. Pese a que su relación con los primeros estudiantes fue cordial, con los últimos resultó compleja. Sáenz de Heredia se marchó de la institución "por una serie de malos estilos, de malos modos, principalmente entre los alumnos"8.

Luis Ponce de León fue nombrado directamente por José María García Escudero quien, años después, reconoció que se equivocó en el nombramiento: "realmente acreditó que se puede ser muy inteligente, y desde el punto de vista doctrinal muy abierto $y$, sin embargo, carecer en absoluto de los dotes de gobierno que exigen una cierta flexibilidad, una cierta accesibilidad" 9 .

También, García Escudero, reconoció su error en la elección de Carlos Fernández Cuenca, que no creó problemas pero no supo resolver los ya existentes. Este no fue capaz de contentar ni al alumnado y ni a la administración. En este momento la Dirección General de Cinematografía pensó en volver a nombrar de nuevo a un profesional y se eligió a Antonio Cuevas, productor y buen conocedor de la industria, previsiblemente capaz de despolitizar el centro y de darle el impulso que le devolviera su significado y su sentido. Sin embargo, no fue eso lo que ocurrió; el conflicto era ya permanente y estructural. Gran parte de los discentes había ya optado por el rechazo a cualquier dependencia de la administración. Tras un corto periodo en el cargo, Cuevas dimitió enfrentado al alumnado y a la Dirección General.

El último director de la escuela fue también el más controvertido de todos. Juan Julio Baena resulta aún hoy, según el recuerdo de los estudiantes, una figura ambivalente e, incluso, oscura. El alumnado le pidió que dimitiera al poco de comenzar su mandato. Sin embargo, él se negó e intentó imponer su criterio a las nuevas genera-

8 Entrevista de Lucio Blanco Mallada a José Luis Sáenz de Heredia el día 15-09-1984.

9 Entrevista de Lucio Blanco Mallada a José María García Escudero el día 07-09-1984. ciones de ingresados. El enfrentamiento, no obstante, se mantuvo y condujo, en última instancia, al cierre de la institución.

\subsection{Breve evolución}

Existían dos tipos de prácticas regladas: las que se hacían para cada especialidad -en Cámara se filmaban prácticas de tiempo de exposición, iluminación, composición, movimientos de cámara...; en Decoración se dibujaban planos y se construían decorados; en Dirección se realizaban ensayos de rodajes...- y las prácticas conjuntas para las que se formaba un equipo compuesto por alumnos de todas las especialidades. Las prácticas de fin de carrera, por las que se obtenía la titulación, eran la culminación de todos estos trabajos conjuntos.

En los primeros años, con un presupuesto anual de 200.000 pesetas, las prácticas eran mucho más limitadas habiendo de ser codirigidas varias de ellas. A finales de los años cincuenta se amplía el presupuesto a 600.000 pesetas. Y las prácticas adquieren otro rango. Aumenta el metraje, son catalogadas y todo el equipo consta en los títulos de crédito. En la última etapa, en la Dehesa de la Villa, el centro cuenta con un presupuesto de 15.000 .000 pesetas. Y las prácticas, consecuentemente, cuentan con más medios y recursos.

Uno de los ejercicios más interesantes fue La tarde del domingo del curso 1956-57 dirigida y escrita por Carlos Saura y fotografiada por Luis Enrique Torán, alumnos que llegaron a ser profesores de la EOC. En la primera parte de esta película se puede apreciar una clara tendencia neorrealista; en la segunda, en cambio, hay un montaje mucho más dinámico, de estilo más moderno. Parecido título tiene la práctica escrita y dirigida por Basilio Martín Patino, Tarde de Domingo, del curso 1960-61, fotografiada por Luis Cuadrado. Construía un tempo lento sobre el cual se hacía una indagación sobre el tedio -el personal y también el social-. Llama la atención la similitud de estos dos títulos, que se debe a su proximidad temática, dando cuenta del escaso aliciente de la vida en ese hastío rutinario de la 
sociedad española de los años cincuenta. Un país con una moral exhausta de la que se desprende ese hálito de muerte que está presente en los dos films ${ }^{10}$. En el mismo curso Francisco Prósper trazó, en clave de comedia y usando de forma creativa la óptica de la cámara, el retrato de un hombre de clase media que se ve obligado aceptar un trabajo que no está a la altura de su supuesta posición social.

En el curso 1958-59 se rueda El viejecito, de Manuel Summers, que incluye los temas más recurrentes en su filmografía: la muerte, sus ritos y su liturgia. También en ese año se filma Habitación de alquiler, dirigida por Miguel Picazo, donde de nuevo aparece la muerte como trasfondo. Un año después, José Luis Borau rueda En el rio, tierna historia de un joven que contempla a una mujer en bikini. El deseo que se presenta en el relato da una muestra de la vida que parece abrirse a un nuevo tiempo, el de los años sesenta.

En los cursos 1959-60 y 1960-61, el despegue económico y su miseria cultural se muestran con claridad en Despedida de soltero, de José Luis Viloria; La cinta, de Horacio Valcárcel, es un film bastante críptico donde se indagaba en la difícil convivencia entre lo puro ideal y lo imperfecto material; y La lágrima del diablo, de Julio Diamante, desarrollaba varios géneros cinematográficos y estaba contada con originalidad y un gran conocimiento del oficio más allá de lo normal en un simple estudiante de cine.

En el curso 1961-62 destacaron tres prácticas: Turno de noche, realizada por Luis Enrique Torán, en la cual se denunciaba el fuerte clasismo social y la existencia de las clases desfavorecidas en aquellos años. La estrategia narrativa de esta práctica, la composición, la dialéctica espacio in/ espacio off y la fotografía de Luis Cuadrado hacen de esta obra una de las más destacadas de la Escuela. Es, desde luego, una de las más alejadas de la influencia del neorrealismo y más cercana al cine de géneros de Hollywood. El borracho,

10 En el sentido que McLuham daba a la palabra, es decir, lo que no se ve pero se percibe, inscrita en su Teoría de la percepción (McLuhan, 2008). de Mario Camus es una amarga película sobre la marginación y el abandono de un alcohólico que cuenta también con una significada fotografía de Luis Cuadrado y con una memorable interpretación de Sergio Mendizábal. Por último, Sor Angélica Virgen, trabajo de Francisco Regueiro y fotografía de Víctor Monreal aborda la religión como pieza clave de la superestructura del régimen franquista.

En el curso 1962-63 la práctica de Víctor Erice Los días perdidos se distancia claramente de las demás. Se trata de un gran cortometraje que solo pierde parte de su fuerza por el exceso de voz en off y por la interpretación de su protagonista masculino. Esta película puede considerarse como una de las mejores filmadas en la Escuela. Antonio Artero dirigió en el curso 1963-64 Doña Rosita la soltera, con una original puesta en escena y no menos original estructura narrativa; un justo tono de comedia que se abandona al final. Interesante también Luciano, de Claudio Guerín Hill, una obra adelantada del realismo mágico. La educación, la represión religiosa-cultural y la manipulación de los hechos por parte de los medios son algunos de los temas más importantes que aborda esta práctica.

La caza de Brujas dirigida por Antonio Drove y Los preparativos de Álvaro del Amo son dos de las más destacadas, ambas en el curso 1966-67. La práctica de Antonio Drove es la más conocida de toda la historia de la EOC, habiendo sido una referencia en cineclubs y en las revistas cinematográficas de aquellos años. En la obra de Álvaro Del Amo se hace notar el oficio de su director, que optó por un cine experimental, estética perfectamente adecuada para la farsa concebida en el guion. Manuel Gutiérrez Aragón realiza Hansel y Gretel en 1968-69. Se anticipa ya en ella la sinergia entre el realismo y la alucinación visionaria que su autor desarrollaría en su filmografía posterior. En el mismo curso Cecilia Bartolomé rueda Margarita y el lobo una obra inteligente y radical sobre las relaciones de pareja.

En conclusión, se hace notar que las prácticas de la EOC no muestran interés en los géneros cinematográficos, más bien se percibe un cierto 
desprecio por el cine de Hollywood. Observemos que cuando aparecen claves de género son utilizadas de forma irónica y como deconstrucción de su código fílmico. La tendencia estilística generalizada es avanzar hacia el cine de autor. Además, frecuentemente las tareas de guion y dirección son asumidas por la misma persona.

Los movimientos cinematográficos y corrientes estéticas de esos años muestran su presencia en las prácticas. Casi la totalidad de las obras de los primeros años muestran una notable influencia neorrealista. En los años sesenta ${ }^{11}$ llega a la Escuela la influencia de la Nouvelle $\mathrm{Va}$ gue y, algo más tarde, el Cinema Novo Brasileiro ${ }^{12}$, que inspira las obras de los guionistas y se hace notar en escenografía e, incluso, en interpretación. La estética de la violencia del Cinema Novo desplaza hacia un segundo término la propia estética para dar mayor importancia a una actitud ética inseparable del compromiso político, exactamente lo que estaba ocurriendo en la EOC en esos años finales de su recorrido.

\section{CONCLUSIONES}

El recorrido entre las Conversaciones de Salamanca en 1955 y el Festival de Sitges en 1967 describe lo acontecido en España en el ámbito cultural en general y, específicamente, en el cinematográfico. Las Conversaciones de Salamanca significan una voluntad de entendimiento entre la industria cinematográfica y el

11 Además, en esta época se suceden una serie de mejoras técnicas que ofrece la institución a la hora de ejecutar las prácticas: "A partir de 1970 la EOC dispone de más medios técnicos y presupuestarios y los alumnos comienzan a rodar en 35 milímetros desde el primer año. Al mismo tiempo aumenta el número de las prácticas por curso, se utiliza el color de forma generalizada y se incorpora, en los trabajos en 16 milímetros, el sonido magnético" (Página web del Ministerio de Educación, Cultura y Deporte: [http://goo.gl/CfKLLP] Consultado el 26/febrero/2016).

12 Las nuevas olas -Nouvelle Vague, Free Cinema, Cinema Novo...- abren otras vías expresivas a los alumnos. Sin embargo, "el objetivo de modernizar el cine español promocionando el trabajo de los jóvenes realizadores resultó un fracaso por las reticencias de casi todos los implicados" (García-Merás, 2007: 21).
IIEC a través de la Dirección General de Cine. Los planteamientos surgidos en el Festival de Sitges supusieron el final del entendimiento en los términos en que este se había establecido y la imposibilidad de acuerdo alguno dentro del arco político del régimen. Incluso estos años supusieron un rechazo total de la estética cinematográfica tal y como se había concebido hasta entonces.

El apartado octavo de las Conversaciones de Salamanca estuvo dedicado específicamente al IIEC con el título de Formación Profesional. Expuesto de un modo sucinto, este apartado decía que el instituto debía ser la fuente principal de la que se nutriera la industria cinematográfica española, por lo que este centro debería ofrecer las máximas garantías para que sus graduados pudieran desempeñar el cometido que correspondiese a sus cargos en los equipos de las películas que se fueran a producir. Para ello era necesario que hubiese una ayuda estatal suficiente para resolver los problemas que existían en aquel momento en la escuela. Además, debería contar con un profesorado competente en cuyo nombramiento intervendría de un modo activo el voto del alumnado. Otras aspectos reseñados eran: máximo rigor en los exámenes de ingreso sin que fuera preciso titulación académica para poder acceder; un plan de estudios suficientemente extenso para permitir la formación eficiente del alumnado; intensificación de las prácticas y que estas se efectúen con todos los medios propios del cine profesional; integración provisional de los alumnos en los equipos de las películas producidas profesionalmente como técnicos adjuntos; concesión del carnet sindical a todos los egresados del IIEC y la integración de este centro en el mismo Ministerio que la Dirección General de Cine.

En un marco más amplio, el régimen franquista no supo dar respuesta a lo que la juventud, especialmente la universitaria, demandaba. Hasta 1955 había sido posible un cierto entendimiento del gobierno con la Universidad. Ese entendimiento se hace imposible a partir de las huelgas estudiantiles de 1956. 
Desde ese momento, la mayoría de los discentes españoles estuvieron ya siempre en contra del régimen.

El intento de apertura de Manuel Fraga Iribarne al frente del Ministerio del Interior y Turismo supone una oportunidad para la Escuela. Así, el nombramiento de José María García Escudero como director ofrece un pacto tácito; una mayor permisividad en los temas y en la forma de exponerlos a cambio de aceptar unos límites. La política de subvenciones y ayudas promovió la etapa de oro de la Escuela y el nacimiento del Nuevo Cine Español. Sin embargo, la llegada de Juan Julio Baena a la dirección del centro supuso un enconamiento entre alumnado y administración. Se rompió definitivamente la posibilidad de un consenso o un acuerdo.

Los alumnos de las primeras promociones del IIEC -Saura, Patino, Camus...- aceptaron los términos de ese acuerdo, sin embargo los de las últimas promociones de la EOC no lo hicieron. Renunciaron a todo tipo de dependencia de la administración y con ello se cierra su acceso al cine profesional y a su incorporación a la industria cinematográfica, que no daba ninguna muestra de interés por sus planteamientos cinematográficos. Estas actitudes condujeron, finalmente, al cierre de la escuela. 


\section{BIBLIOGRAFÍA}

BLANCO MALLADA, Lucio. IIEC y EOC: Una escuela para el cine español, Universidad Complutense de Madrid, Madrid, 1990.

ARANZUBIA COB, Asier y CASTRO DE PAZ, José Luis. "Desmontando el discurso televisivo: Luciano (Claudio Guerín Hill, 1964-1965)", Zer, Volumen 15, Num. 29, 2010, pp. 13-30.

DELTELL, Luis. "Antonio Lara director de cine", en GARCÍA-FERNÁNDEZ, Emilio C. (Coordinador). Antonio Lara, la enseñanza de la imagen, Departamento de Comunicación Audiovisual y Publicidad 1, Madrid, 2009.

DELTELL, Luis. "Boris (1966) y El perro (1977). Estudio de dos guiones escritos por Juan Antonio Porto", Área Abierta, vol. 14, n01, marzo 2014.

DELTELL, Luis. "La mujer como sujeto: Josefina Molina en la Escuela Oficial de Cine". SIGNA. Revista de la asociación española de semiótica, Vol. 24, 2015.

GARCÍA-MERÁS, Lydia. "El cine de la disidencia. La producción militante antifranquista (1967-1981)", Desencuentros, 2007, vol. 4, pp. 16-41.

GÓMEZ, Juan Pedro. El cine: una guía de iniciación, Universidad de Murcia, Murcia, 2002.

GUBERN, Román y FONT, Domènec. Un cine para el cadalso, Editorial Euros, Barcelona, 1975.

GUBERN, Román; MONTERDE, José Enrique; PÉREZ PERUCHA, Julio; RIAMBAU, Esteve y TORREIRO, Casimiro. Historia del Cine Español, Cátedra, Madrid, 1995

MCLUHAN, Marshall. Comprender los medios de comunicación. Las extensiones del ser humano, Paidós, Barcelona, 2008.

RODRÍGUEZ MERCHÁN, Eduardo. "La enseñanza del cine en España: perspectiva histórica y panorama actual", Comunicar, vol. 29, 2007.

POZO, Santiago. La industria del cine en España: legislación y aspectos económicos, 1896-1970, Edicions Universitat de Barcelona, Barcelona, 1984.

SÁNCHEZ BIOSCA, Vicente. "Las culturas del tardofranquismo", Ayer, Ediciones de historia, S. A, Madrid, 2007.

\section{ENTREVISTAS INÉDITAS}

GARCÍA ESCUDERO, José María. Entrevista concedida al autor del artículo para su tesis de doctorado "IIEC y EOC Una escuela para el cine español" el día 7-09-1984.

MARTÍN PATINO, Basilio. Entrevista concedida al autor del artículo para su tesis de doctorado "IIEC y EOC Una escuela para el cine español" el día 07-10-1984.
REVUELTA MORENO, Manuel. Entrevista concedida al autor del artículo para su tesis de doctorado "IIEC y EOC Una escuela para el cine español" el día 12-05-1985

SÁNCHEZ DRAGÓ, Fernando. Entrevista concedida al autor del artículo para su tesis de doctorado "IIEC y EOC Una escuela para el cine español" el día 21-08-1985.

SÁENZ DE HEREDIA, José Luis. Entrevista concedida al autor del artículo para su tesis de doctorado "IIEC y EOC Una escuela para el cine español" el día 15-09-1984.

\section{WEBGRAFÍA}

Página web del Ministerio de Educación, Cultura y Deporte: [http://goo.gl/CfKLLP] Consultado el 26/ febrero/2016. 